\title{
Probing the Size-Induced Electronic Structures of CdSe Quantum Dots
}

\author{
Ajay S. Karakoti, Shail P. Sanghavi, P. Nachimuthu, Ping Yang, V. Shutthanandan, Louis J. Terminello \\ and S. Thevuthasan \\ Pacific Northwest National Laboratory, Richland, WA 99352
}

The structural, optical and electronic properties of CdSe quantum dots have been studied for various applications ranging from biomedical imaging (1) and sensing (2) to hybrid solar cells (3). When the size of the nanocrystals decreases below the exciton Bohr's radius, the energy levels become discrete (quantized). Consequently, the energy difference between the levels increases with decreasing the size of the quantum dots. This is inherently determined by the electronic structures of these quantum dots. In addition, these CdSe quantum dots are also known to exhibit a phase transition from a stable hexagonal phase in larger dots to a metastable cubic phase at smaller dot sizes. As the size of quantum dot decreases, the number of surface atoms and the energy associated with that surface increase leading to creation of vacancies, which results in the non-stoichiometric CdSe. However, the mechanism is not clear at atomic level. Therefore, we took a systematic approach to study the size-induced structural, optical and electronic properties of CdSe quantum dots in toluene and drop-casted on Si by various in-situ and ex-situ imaging, spectroscopy and diffraction techniques to obtain the correlation between the quantum confinement and the corresponding stoichiometry, crystalline phases and the effect of surface ligands.

Preliminary UV-Visible absorption and fluorescence studies of the as-synthesized CdSe quantum dots capped with hexadecylamine (HDA) and/or HDA/trioctylphosphine oxide (TOPO) in toluene show an increase in optical density and fluorescence yield with decreasing quantum dot size for the isoconcentration solutions within the experimental error limits (Figure 1(a-b)). The deep trap emission at longer wavelengths arising from the Se vacancies also increases with decreasing quantum dot size. The crystallite size, the optical band gap and the Stoke's shift obtained from the absorption and the fluorescence data are in the range of 2.0-5.5 nm, 1.9-2.5 eV and 15-25 nm, respectively. The photoemission studies of these as-synthesized CdSe quantum dots capped with HDA and/or HDA/TOPO in toluene drop-casted on clean Si wafer show that the $\mathrm{Cd} 3 \mathrm{~d}$ and Se 3d photoelectron peaks shift towards higher binding energy with decreasing quantum dot size which are consistent with quantum confinement effect. The band gap determined from valence band photoelectron spectra shows values similar to that observed from the optical absorption spectra validating the observations. The Se/Cd ratio obtained from the elemental quantification is 0.71 at size of $5.5 \mathrm{~nm}$ and it decreases to 0.63 with decreasing quantum dot size suggesting the increase of CdSe non-stoichiometry. Even though quantum dots are Se deficient, the angle-resolved data show that Se tends to stay on the surface rather than the bulk of the quantum dot. This is also consistent with deep trap emission from the Se vacancies observed at longer wavelengths with decreasing the quantum dot size. As quantum dot size decrease, the $\mathrm{C}$ content and the $\mathrm{C} / \mathrm{N}$ ratio along with the overall surface area of the CdSe quantum dots show an increase suggesting that the number of the HDA capping ligands on the surface changes as a function of quantum dots size and the solvent molecules are increasingly present on the surface. The $\mu$-XRD results from the CdSe quantum dots in toluene loaded onto a capillary and drop-casted on clean Si wafer both of which exhibit hexagonal and cubic phases. The ratio of these phases from CdSe quantum dots in toluene does not change significantly with size. In contrast the $\mu$-XRD data from CdSe quantum dots drop-casted on Si show an increase of cubic phase with decreasing the quantum dot size. The confinement of CdSe quantum dots by the capping ligands remains intact in toluene even upon decreasing the quantum dot size and the coexistence of two phases is most likely arising from the non-stoichiometry of CdSe. In the case of CdSe quantum dots drop- 
casted on $\mathrm{Si}$, the change in phase ratios with decreasing quantum dot size is probably induced from the drying process in addition to the increase of CdSe non-stoichiometry.

In order to understand these phase changes, the morphology including the capping ligands and their distribution on CdSe with respect to the quantum dot size, high-resolution TEM studies on the assynthesized quantum dots have been carried out and the results were correlated with the data from Helium ion microscopy and scanning transmission x-ray microscopy (STXM) studies. Helium ion microscopy provides better depth-of-view and phase contrast especially for low Z elements which facilitate understanding the distribution of the capping ligands on CdSe. Also the STXM provides both elementalspecific mapping and spectroscopy with a spatial resolution of $20 \mathrm{~nm}$ or better. These preliminary investigation shows that the HDA capping ligands and to a lesser degree the solvent molecules around the CdSe play significant role in determining the structural and electronic properties of these quantum dots.

\section{References:}

1. X. Michalet et al Science, 2005, 307, 538-544.

2. Igor L. Medintz et al Nature Materials, 2005, 4, 435-446.

3. S. Guenes and N.S. Saricifti, Inorganica Chimica Acta, 2008, 361, 581-588.
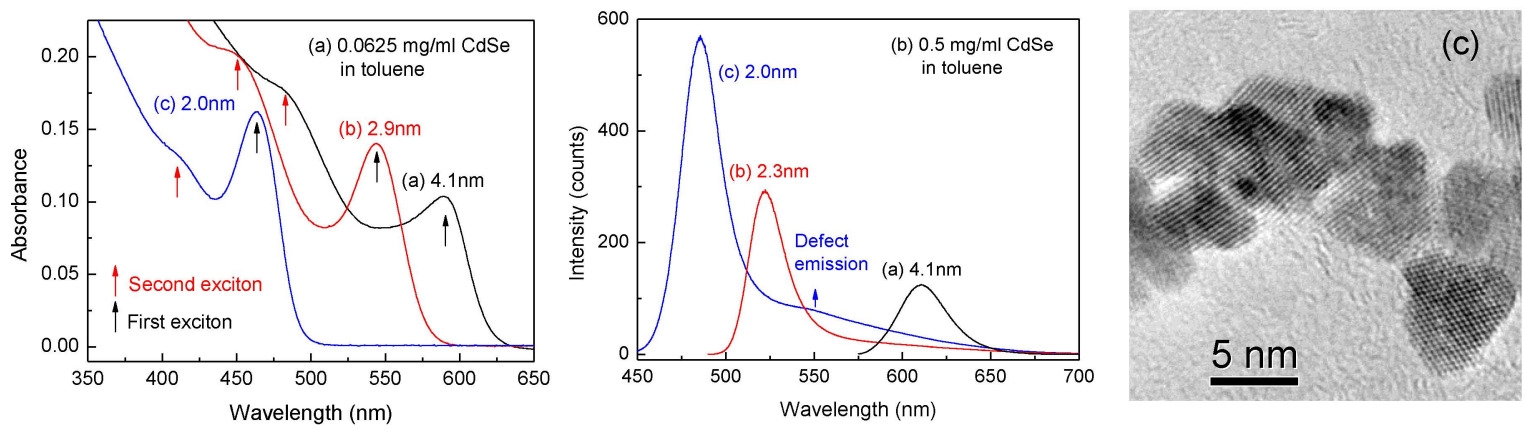

Figure 1: Optical absorption (a) and emission (b) spectra from iso-concentration CdSe quantum dots in toluene showing a decrease in optical density and fluorescence yield with increasing particle size. (c) High resolution transmission electron micrograph from $2.0 \mathrm{~nm}$ sample.
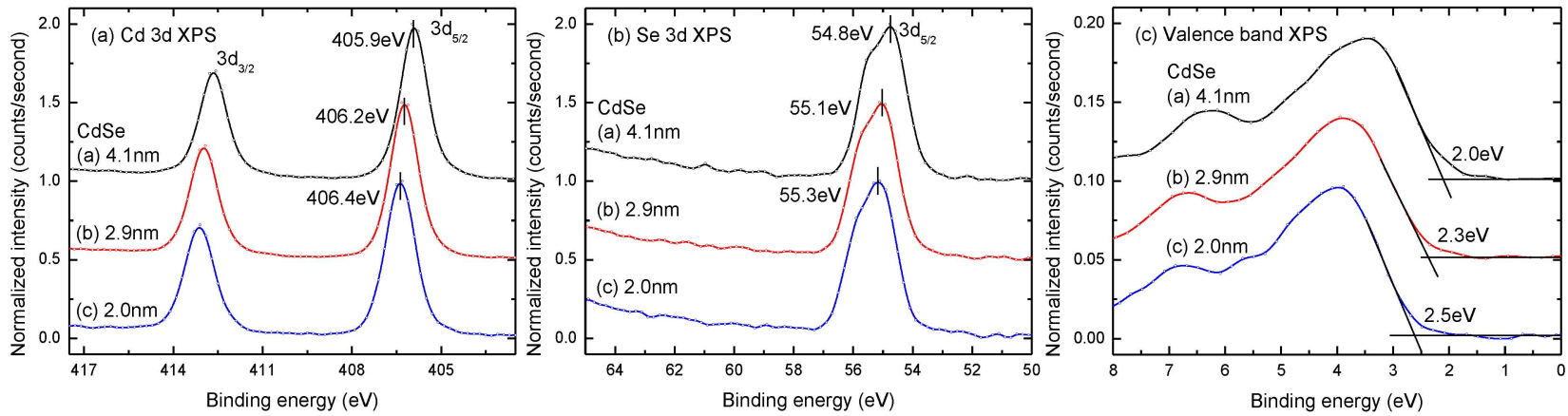

Figure 2: The (a) Cd 3d, (b) Se 3d and (c) valence band XPS from CdSe quantum dots in toluene dropcasted on Si showing size-induced binding energy shifts and the band gaps. 